Belle Preprint 2005-13

KEK Preprint 2005-4

\title{
Study of the Baryon-Antibaryon Low-Mass Enhancements in Charmless Three-body Baryonic $B$ Decays
}

\section{Belle Collaboration}

M.-Z. Wang ${ }^{\mathrm{w}}, \mathrm{K}$. Abe $^{\mathrm{g}}, \mathrm{K}$. Abe ${ }^{\mathrm{a} \ell}, \mathrm{H}$. Aihara ${ }^{\text {an }}$, Y. Asano ${ }^{\text {ar }}$, V. Aulchenko ${ }^{\text {a }}$, T. Aushev $^{\mathrm{k}}$, S. Bahinipati ${ }^{\mathrm{d}}$, A. M. Bakich ${ }^{\text {ai }}$,

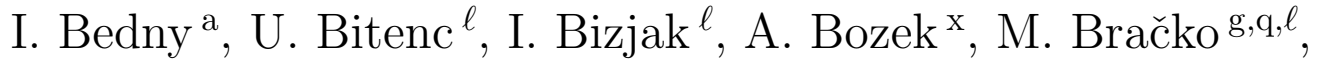
J. Brodzicka ${ }^{\mathrm{x}}$, T. E. Browder ${ }^{\mathrm{f}}$, M.-C. Chang ${ }^{\mathrm{w}}$, P. Chang ${ }^{\mathrm{w}}$, Y. Chao ${ }^{\text {w }}$, A. Chen ${ }^{\text {u }}$, K.-F. Chen ${ }^{\mathrm{w}}$, W. T. Chen ${ }^{\mathrm{u}}$, B. G. Cheon ${ }^{\text {c }}$, R. Chistov ${ }^{\mathrm{k}}$, S.-K. Choi ${ }^{\mathrm{e}}$, A. Chuvikov ${ }^{\text {ae }}$, S. Cole ${ }^{\text {ai }}$, J. Dalseno ${ }^{\mathrm{r}}$, M. Danilov ${ }^{\mathrm{k}}$, M. Dash $^{\text {as }}$, A. Drutskoy ${ }^{\mathrm{d}}$, S. Eidelman ${ }^{\text {a }}$, Y. Enari ${ }^{\mathrm{s}}$, F. Fang ${ }^{\mathrm{f}}$, S. Fratina ${ }^{\ell}$, N. Gabyshev ${ }^{\mathrm{a}}$, T. Gershon ${ }^{\mathrm{g}}$, G. Gokhroo ${ }^{\mathrm{aj}}$, B. Golob ${ }^{\mathrm{p}, \ell}$, A. Gorišek ${ }^{\ell}$,

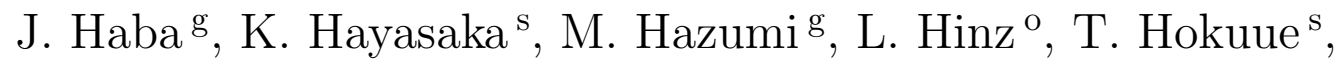
Y. Hoshi ${ }^{a}$, S. Hou ${ }^{\text {u }}$, W.-S. Hou ${ }^{\text {w }}$, Y. B. Hsiung ${ }^{\text {w }}$, T. Iijima ${ }^{\mathrm{s}}$, A. Imoto ${ }^{t}, \mathrm{~K}$. Inami ${ }^{\mathrm{s}}$, A. Ishikawa ${ }^{\mathrm{g}}, \mathrm{R}$. Itoh $^{\mathrm{g}}, \mathrm{M}$. Iwasaki ${ }^{\text {an }}$, Y. Iwasaki ${ }^{g}$, J. H. Kang ${ }^{\text {at }}$, J. S. Kang ${ }^{\mathrm{m}}$, N. Katayama ${ }^{\mathrm{g}}$, H. Kawai ${ }^{\text {b }}$, T. Kawasaki ${ }^{\text {z }}$, H. R. Khan ${ }^{\text {ao }}$, H. Kichimi ${ }^{\mathrm{g}}$, H. J. Kim ${ }^{n}$, S. K. Kim ${ }^{\text {ag }}$, S. M. Kim ${ }^{\text {ah }}$, K. Kinoshita ${ }^{d}$, S. Korpar ${ }^{\mathrm{q}, \ell}$, P. Križan ${ }^{\mathrm{p}, \ell}$, P. Krokovny ${ }^{\mathrm{a}}$, S. Kumar ${ }^{\text {ac }}$, C. C. Kuo ${ }^{u}$, T.-L. Kuo ${ }^{w}$, A. Kuzmin ${ }^{\text {a }}$, Y.-J. Kwon ${ }^{\text {at }}$, G. Leder ${ }^{j}$, S. E. Lee ${ }^{\text {ag }}$, Y.-J. Lee ${ }^{w}$, T. Lesiak ${ }^{\mathrm{x}}$, J. Li $^{\text {af }}$, S.-W. Lin ${ }^{\mathrm{w}}$, D. Liventsev ${ }^{\mathrm{k}}$, J. MacNaughton ${ }^{\mathrm{j}}$, F. Mandl $^{\mathrm{j}}$, T. Matsumoto $^{\text {ap }}$, A. Matyja ${ }^{\mathrm{x}}$, W. Mitaroff ${ }^{\mathrm{j}}$, H. Miyake $^{\mathrm{ab}}$, H. Miyata ${ }^{\mathrm{z}}$, R. Mizuk ${ }^{\mathrm{k}}$, G. R. Moloney ${ }^{\mathrm{r}}$, T. Nagamine ${ }^{\mathrm{am}}$, Y. Nagasaka ${ }^{\text {h}}$, E. Nakano ${ }^{\text {aa }}$, M. Nakao ${ }^{g}$, H. Nakazawa ${ }^{g}$, Z. Natkaniec ${ }^{\mathrm{x}}$, S. Nishida ${ }^{\mathrm{g}}$, O. Nitoh ${ }^{\mathrm{aq}}$, S. Ogawa ${ }^{\mathrm{ak}}$, T. Ohshima ${ }^{\text {s, T. Okabe }}{ }^{\text {s }, ~ S . ~ L . ~ O l s e n ~}{ }^{\text {f }}$, W. Ostrowicz ${ }^{\mathrm{x}}$, H. Ozaki ${ }^{\mathrm{g}}, \mathrm{H}$. Palka ${ }^{\mathrm{x}}$, C. W. Park ${ }^{\text {ah }}$, N. Parslow ${ }^{\text {ai }}$, L. S. Peak ${ }^{\text {ai }}$, R. Pestotnik ${ }^{\ell}$, L. E. Piilonen ${ }^{\text {as }}$, N. Root ${ }^{\text {a }}$, H. Sagawa ${ }^{g}$, Y. Sakai ${ }^{g}$, N. Sato ${ }^{\text {s }}$, T. Schietinger ${ }^{\text {, }}$, 
O. Schneider ${ }^{\text {}}$, J. Schümann ${ }^{\mathrm{w}}$, K. Senyo ${ }^{\mathrm{s}}$, M. E. Sevior ${ }^{\mathrm{r}}$, T. Shibata ${ }^{\text {, H. H. Shibuya }}{ }^{\text {ak }}$, B. Shwartz ${ }^{\text {a }}$, V. Sidorov ${ }^{\mathrm{a}}$,

J. B. Singh ${ }^{\text {ac }}$, A. Somov ${ }^{\text {d }}$, N. Soni ${ }^{\text {ac }}$, R. Stamen ${ }^{\mathrm{g}}$, S. Stanič ${ }^{\text {ar, }}{ }^{\text {, }}$ M. Starič ${ }^{\ell}$, K. Sumisawa ${ }^{\text {ab }}$, T. Sumiyoshi ${ }^{\text {ap }}$, O. Tajima ${ }^{g}$, F. Takasaki ${ }^{\mathrm{g}}$, K. Tamai ${ }^{\mathrm{g}}$, N. Tamura ${ }^{\mathrm{z}}$, M. Tanaka ${ }^{\mathrm{g}}$, Y. Teramoto ${ }^{\text {aa }}$, X. C. Tian ${ }^{\text {ad }}$, T. Tsukamoto ${ }^{g}$, S. Uehara $^{\mathrm{g}}$, K. Ueno ${ }^{\text {w }}$, T. Uglov ${ }^{\mathrm{k}}$, S. Uno ${ }^{\mathrm{g}}$, P. Urquijo ${ }^{\mathrm{r}}$, G. Varner ${ }^{\mathrm{f}}$, K. E. Varvell ${ }^{\text {ai }}$, S. Villa ${ }^{\circ}$, C. C. Wang ${ }^{w}$, C. H. Wang ${ }^{\mathrm{v}}$, M. Watanabe ${ }^{\text {, }, ~ Q . ~ L . ~ X i e ~}{ }^{\text {i }, ~ B . ~ D . ~ Y a b s l e y ~}{ }^{\text {as }}$, A. Yamaguchi ${ }^{\text {am }}$, Y. Yamashita ${ }^{\mathrm{y}}$, M. Yamauchi ${ }^{\mathrm{g}}$, Heyoung Yang ${ }^{\mathrm{ag}}$, J. Ying ad, L. M. Zhang af ${ }^{\text {, Z. P. Zhang }}{ }^{\text {af }}$, V. Zhilich ${ }^{\mathrm{a}}$, D. Žntar ${ }^{\mathrm{p}, \ell}$ and

${ }^{a}$ Budker Institute of Nuclear Physics, Novosibirsk, Russia

${ }^{\mathrm{b}}$ Chiba University, Chiba, Japan

${ }^{\mathrm{c}}$ Chonnam National University, Kwangju, South Korea

${ }^{\mathrm{d}}$ University of Cincinnati, Cincinnati, OH, USA

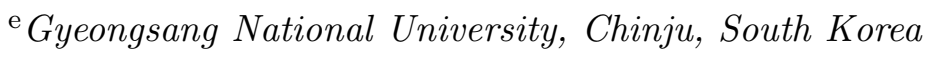

${ }^{\mathrm{f}}$ University of Hawaii, Honolulu, HI, USA

${ }^{\mathrm{g}}$ High Energy Accelerator Research Organization (KEK), Tsukuba, Japan

${ }^{\mathrm{h}}$ Hiroshima Institute of Technology, Hiroshima, Japan

${ }^{\mathrm{i}}$ Institute of High Energy Physics, Chinese Academy of Sciences, Beijing, PR China

${ }^{\mathrm{j}}$ Institute of High Energy Physics, Vienna, Austria

${ }^{\mathrm{k}}$ Institute for Theoretical and Experimental Physics, Moscow, Russia

${ }^{\ell} J$. Stefan Institute, Ljubljana, Slovenia

${ }^{\mathrm{m}}$ Korea University, Seoul, South Korea

${ }^{\mathrm{n}}$ Kyungpook National University, Taegu, South Korea

${ }^{\circ}$ Swiss Federal Institute of Technology of Lausanne, EPFL, Lausanne, Switzerland

${ }^{\mathrm{p}}$ University of Ljubljana, Ljubljana, Slovenia

${ }^{\mathrm{q}}$ University of Maribor, Maribor, Slovenia

${ }^{\mathrm{r}}$ University of Melbourne, Victoria, Australia

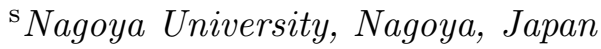

' Nara Women's University, Nara, Japan

u National Central University, Chung-li, Taiwan

${ }^{\mathrm{v}}$ National United University, Miao Li, Taiwan

${ }^{\mathrm{w}}$ Department of Physics, National Taiwan University, Taipei, Taiwan

${ }^{\mathrm{x}}$ H. Niewodniczanski Institute of Nuclear Physics, Krakow, Poland 
${ }^{\mathrm{y}}$ Nihon Dental College, Niigata, Japan

${ }^{\mathrm{z}}$ Niigata University, Niigata, Japan

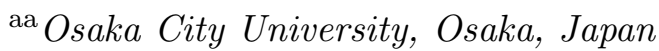

${ }^{\mathrm{ab}}$ Osaka University, Osaka, Japan

ac Panjab University, Chandigarh, India

ad Peking University, Beijing, PR China

ae Princeton University, Princeton, NJ, USA

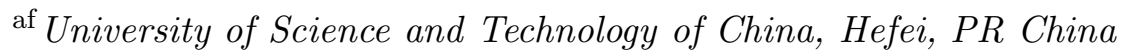

${ }^{\text {ag }}$ Seoul National University, Seoul, South Korea

${ }^{\text {ah }}$ Sungkyunkwan University, Suwon, South Korea

${ }^{\text {ai }}$ University of Sydney, Sydney, NSW, Australia

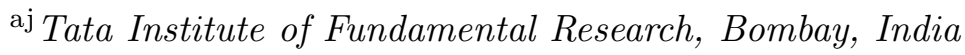

${ }^{\text {ak }}$ Toho University, Funabashi, Japan

al Tohoku Gakuin University, Tagajo, Japan

am Tohoku University, Sendai, Japan

an Department of Physics, University of Tokyo, Tokyo, Japan

ao Tokyo Institute of Technology, Tokyo, Japan

ap Tokyo Metropolitan University, Tokyo, Japan

${ }^{a q}$ Tokyo University of Agriculture and Technology, Tokyo, Japan

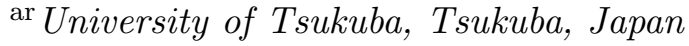

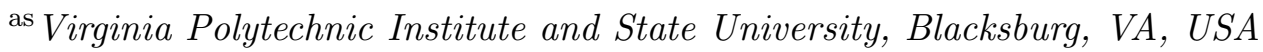

at Yonsei University, Seoul, South Korea

\begin{abstract}
The angular distributions of the baryon-antibaryon low-mass enhancements seen in the charmless three-body baryonic $B$ decays $B^{+} \rightarrow p \bar{p} K^{+}, B^{0} \rightarrow p \bar{p} K_{S}^{0}$, and $B^{0} \rightarrow$ $p \bar{\Lambda} \pi^{-}$are reported. A quark fragmentation interpretation is supported, while the gluonic resonance picture is disfavored. Searches for the $\Theta^{+}$and $\Theta^{++}$pentaquarks in the relevant decay modes and possible glueball states $\mathcal{G}$ with $2.2 \mathrm{GeV} / c^{2}<$ $M_{p \bar{p}}<2.4 \mathrm{GeV} / c^{2}$ in the $p \bar{p}$ systems give null results. We set upper limits on the products of branching fractions, $\mathcal{B}\left(B^{0} \rightarrow \Theta^{+} \bar{p}\right) \times \mathcal{B}\left(\Theta^{+} \rightarrow p K_{S}^{0}\right)<2.3 \times 10^{-7}$, $\mathcal{B}\left(B^{+} \rightarrow \Theta^{++} \bar{p}\right) \times \mathcal{B}\left(\Theta^{++} \rightarrow p K^{+}\right)<9.1 \times 10^{-8}$, and $\mathcal{B}\left(B^{+} \rightarrow \mathcal{G} K^{+}\right) \times \mathcal{B}(\mathcal{G} \rightarrow$ $p \bar{p})<4.1 \times 10^{-7}$ at the $90 \%$ confidence level. The analysis is based on a $140 \mathrm{fb}^{-1}$ data sample recorded on the $\Upsilon(4 \mathrm{~S})$ resonance with the Belle detector at the KEKB asymmetric-energy $e^{+} e^{-}$collider.
\end{abstract}

PACS: $13.25 . \mathrm{Hw}, 13.60 . \mathrm{Rj}$ 
Observations of several baryonic three-body $B$ decays have been reported recently $[1,2,3,4]$. One common feature of these observations is the peaking of the baryon-antibaryon pair mass spectra toward threshold, as originally conjectured in Refs. [5,6] and elaborated more recently in Refs. [7,8,9]. The same peaking behavior near threshold has been found in baryonic $J / \psi$ decays [10] as well, indicating that this may be a universal phenomenon. Possible explanations include intermediate (gluonic) resonant states or non-perturbative QCD effects of the quark fragmentation process $[7,8]$. Alternatively, the dynamical picture can be replaced by an effective range analysis with a baryon form factor [9]. To distinguish among the above hypotheses for the production mechanism, we study the angular distributions of the threshold enhancements in the helicity frame for the $B^{+} \rightarrow p \bar{p} K^{+}, B^{0} \rightarrow p \bar{p} K_{S}^{0}$ and $B^{0} \rightarrow p \bar{\Lambda} \pi^{-}[11]$ modes.

We use a $140 \mathrm{fb}^{-1}$ data sample, consisting of $152 \times 10^{6} B \bar{B}$ pairs, collected with the Belle detector at the KEKB asymmetric energy $e^{+} e^{-}(3.5$ on $8 \mathrm{GeV})$ collider [12]. The Belle detector is a large solid angle magnetic spectrometer that consists of a three layer silicon vertex detector (SVD), a 50 layer central drift chamber (CDC), an array of aerogel threshold Cerenkov counters (ACC), a barrel-like arrangement of time of flight scintillation counters (TOF), and an electromagnetic calorimeter comprised of $\mathrm{CsI}(\mathrm{Tl})$ crystals located inside a superconducting solenoid coil that provides a $1.5 \mathrm{~T}$ magnetic field. An iron flux return located outside of the coil is instrumented to detect $K_{L}^{0}$ mesons and to identify muons. The detector is described in detail elsewhere [13].

The event selection criteria are based on the information obtained from the tracking system (SVD and CDC) and the hadron identification system (CDC, ACC, and TOF). All primary charged tracks are required to satisfy track quality criteria based on the track impact parameters relative to the interaction point (IP). The deviations from the IP position are required to be within $\pm 1 \mathrm{~cm}$ in the transverse $(x-y)$ plane, and within $\pm 3 \mathrm{~cm}$ in the $z$ direction, where the $z$ axis is opposite the positron beam direction. For each track, the likelihood values $L_{p}, L_{K}$, and $L_{\pi}$ that it is a proton, kaon, or pion, respectively, are determined from the information provided by the hadron identification system. The track is identified as a proton if $L_{p} /\left(L_{p}+L_{K}\right)>0.6$ and $L_{p} /\left(L_{p}+L_{\pi}\right)>0.6$, or as a kaon if $L_{K} /\left(L_{K}+L_{\pi}\right)>0.6$, or as a pion if $L_{\pi} /\left(L_{K}+L_{\pi}\right)>0.6$. The proton selection efficiency is about $84 \%(88 \%$ for $p$ and $80 \%$ for $\bar{p}$ ) for particles with momenta at $2 \mathrm{GeV} / c$, and the fake rate is about $10 \%$ for kaons and $3 \%$ for pions. Candidate $K_{S}^{0}$ mesons are reconstructed from pairs of oppositely charged tracks (both treated as pions) having a mass consistent with the $K_{S}^{0}$ nominal mass, $\left|M_{\pi^{+} \pi^{-}}-M_{K^{0}}\right|<30$ $\mathrm{MeV} / c^{2}$, as well as a displaced vertex and flight direction consistent with an origin at the IP. Candidate $\Lambda$ baryons are reconstructed from pairs of oppo-

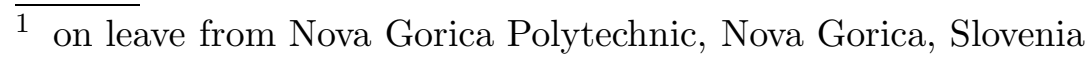


sitely charged tracks - treated as a proton and negative pion-whose mass is consistent with the nominal $\Lambda$ baryon mass, $1.111 \mathrm{GeV} / c^{2}<M_{p \pi^{-}}<1.121$ $\mathrm{GeV} / c^{2}$. The proton-like daughter is required to satisfy $L_{p} /\left(L_{p}+L_{\pi}\right)>0.6$.

Candidate $B$ mesons are reconstructed in the $B^{+} \rightarrow p \bar{p} K^{+}, B^{0} \rightarrow p \bar{p} K_{S}^{0}$, and $B^{0} \rightarrow p \bar{\Lambda} \pi^{-}$modes. We use two kinematic variables in the center of mass $(\mathrm{CM})$ frame to identify the reconstructed $B$ meson candidates: the beam energy constrained mass $M_{\mathrm{bc}}=\sqrt{E_{\mathrm{beam}}^{2}-p_{B}^{2}}$, and the energy difference $\Delta E=$ $E_{B}-E_{\text {beam }}$, where $E_{\text {beam }}$ is the beam energy, and $p_{B}$ and $E_{B}$ are the momentum and energy, respectively, of the reconstructed $B$ meson. The candidate region is defined as $5.20 \mathrm{GeV} / c^{2}<M_{\mathrm{bc}}<5.29 \mathrm{GeV} / c^{2}$ and $-0.1 \mathrm{GeV}<\Delta E<0.2$ $\mathrm{GeV}$. From a GEANT [14] based Monte Carlo (MC) simulation, the signal peaks in the subregion $5.27 \mathrm{GeV} / c^{2}<M_{\mathrm{bc}}<5.29 \mathrm{GeV} / c^{2}$ and $|\Delta E|<0.05$ $\mathrm{GeV}$. The lower bound of $\Delta E$ is chosen to exclude possible contamination from so-called "cross-feed" baryonic $B$ decays.

The background in the fit region arises solely from the continuum $e^{+} e^{-} \rightarrow q \bar{q}$ $(q=u, d, s, c)$ process. We suppress the jet-like continuum background events relative to the more spherical $B \bar{B}$ signal events using a Fisher discriminant [15] that combines seven event shape variables, as described in Ref. [16]. Probability density functions (PDFs) for the Fisher discriminant and the cosine of the angle between the $B$ flight direction and the beam direction in the $\Upsilon(4 \mathrm{~S})$ rest frame are combined to form the signal (background) likelihood $\mathcal{L}_{s}$ $\left(\mathcal{L}_{b}\right)$. The signal PDFs are determined using signal MC simulation; the background PDFs are obtained from the side-band data with $M_{\mathrm{bc}}<5.26 \mathrm{GeV} / c^{2}$. We require the likelihood ratio $\mathcal{R}=\mathcal{L}_{s} /\left(\mathcal{L}_{s}+\mathcal{L}_{b}\right)$ to be greater than $0.7,0.75$, and 0.8 for the $p \bar{p} K^{+}, p \bar{p} K_{S}^{0}$, and $p \bar{\Lambda} \pi^{-}$modes, respectively. These selection criteria are determined by optimization of $n_{s} / \sqrt{n_{s}+n_{b}}$, where $n_{s}$ and $n_{b}$ denote the expected numbers of signal and background events, respectively. We use the branching fractions from our previous measurements $[2,3]$ in the calculation of $n_{s}$. If there are multiple $B$ candidates in a single event, we select the one with the best $\chi^{2}$ value from the vertex fit.

We perform an unbinned likelihood fit that maximizes the likelihood function,

$$
L=\frac{e^{-\left(N_{s}+N_{b}\right)}}{N !} \prod_{i=1}^{N}\left[N_{s} P_{s}\left(M_{\mathrm{bc}_{i}}, \Delta E_{i}\right)+N_{b} P_{b}\left(M_{\mathrm{bc}_{i}}, \Delta E_{i}\right)\right]
$$

to estimate the signal yield in $5.20 \mathrm{GeV} / c^{2}<M_{\mathrm{bc}}<5.29 \mathrm{GeV} / c^{2}$ and -0.1 $\mathrm{GeV}<\Delta E<0.2 \mathrm{GeV}$; here $P_{s}\left(P_{b}\right)$ denotes the signal (background) PDF, $N$ is the number of events in the fit, and $N_{s}$ and $N_{b}$ are fit parameters representing the number of signal and background events, respectively.

For the signal PDF, we use the product of a Gaussian in $M_{\mathrm{bc}}$ and a double Gaussian in $\Delta E$. We fix the parameters of these functions to values determined 
by MC simulation [17]. The continuum background PDF is taken as the product of shapes in $M_{\mathrm{bc}}$ and $\Delta E$, which are assumed to be uncorrelated. We use the parameterization first used by the ARGUS collaboration [18], $f\left(M_{\mathrm{bc}}\right) \propto M_{\mathrm{bc}} \sqrt{1-x^{2}} \exp \left[-\xi\left(1-x^{2}\right)\right]$, to model the $M_{\mathrm{bc}}$ background, with $x$ given by $M_{\mathrm{bc}} / E_{\mathrm{beam}}$ and $\xi$ as a fit parameter. The $\Delta E$ background shape is modeled by a linear function whose slope is a fit parameter.

The differential branching fraction as a function of the baryon pair mass is shown in Fig. 1. The error bars include the statistical uncertainty from the fit and the systematic uncertainty. Here, the efficiency as a function of baryon pair mass for each signal mode is determined by MC simulation. We sum these partial branching fractions to obtain: $\mathcal{B}\left(B^{+} \rightarrow p \bar{p} K^{+}\right)=$ $\left(5.30_{-0.39}^{+0.45} \pm 0.58\right) \times 10^{-6}, \mathcal{B}\left(B^{0} \rightarrow p \bar{p} K_{S}^{0}\right)=\left(1.20_{-0.22}^{+0.32} \pm 0.14\right) \times 10^{-6}$, and $\mathcal{B}\left(B^{0} \rightarrow p \bar{\Lambda} \pi^{-}\right)=\left(3.27_{-0.51}^{+0.62} \pm 0.39\right) \times 10^{-6}$ which are in good agreement with previous measurements $[1,2,3]$. These results also supersede our previous measurements with better accuracy. Note that we have imposed a charmonium veto for the $p \bar{p} K^{+}$and $p \bar{p} K_{S}^{0}$ modes: the regions $2.850 \mathrm{GeV} / c^{2}<M_{p \bar{p}}<3.128$ $\mathrm{GeV} / c^{2}$ and $3.315 \mathrm{GeV} / c^{2}<M_{p \bar{p}}<3.735 \mathrm{GeV} / c^{2}$ are excluded to remove background from $B$ decay modes containing an $\eta_{c}, J / \psi, \psi^{\prime}, \chi_{c 0}$, or $\chi_{c 1}$ meson. The width of the low mass enhancement in each distribution of Fig. 1 depends on the signal mode. A narrow width is also observed in the newly discovered $B^{+} \rightarrow \Lambda \bar{\Lambda} K^{+}$decay [4].

Systematic uncertainties are determined using high statistics control data samples. For proton identification, we use a $\Lambda \rightarrow p \pi^{-}$sample, while for $K / \pi$ identification we use a $D^{*+} \rightarrow D^{0} \pi^{+}, D^{0} \rightarrow K^{-} \pi^{+}$sample. Tracking efficiency is measured with fully and partially reconstructed $D^{*}$ samples. The uncertainty of $K_{S}^{0}$ reconstruction due to off-IP tracks is determined from a $D^{-} \rightarrow K_{S}^{0} \pi^{-}$sample. The $\Lambda$ and $K_{S}^{0}$ reconstruction efficiencies have the same uncertainty due to off-IP tracks if the uncertainty of the daughter proton identification criterion is not taken into account. The $\mathcal{R}$ continuum suppression uncertainty is estimated from control samples with similar final states, for example, $B^{+} \rightarrow J / \psi K^{+}$with $J / \psi \rightarrow \mu^{+} \mu^{-}$. Based on these studies, we assign a $1 \%$ error for each track, $3 \%$ for each proton identification, $2 \%$ for each kaon/pion identification, $5 \%$ for $K_{S}^{0}$ and $\Lambda$ off-IP reconstruction and $6 \%$ for the $\mathcal{R}$ selection.

A systematic uncertainty of $4 \%$ in the fit yield is determined by varying the parameters of the signal and background PDFs. The MC statistical uncertainty contributes a $2 \%$ error in the branching fraction determination. The error on the number of $B \bar{B}$ pairs is $0.5 \%$, where we assume that the branching fractions of $\Upsilon(4 \mathrm{~S})$ to neutral and charged $B \bar{B}$ pairs are equal.

We first sum the correlated errors linearly and then combine them with the uncorrelated ones in quadrature. The total systematic uncertainties are $11 \%$, 
$12 \%$, and $12 \%$ for the $p \bar{p} K^{+}, p \bar{p} K_{S}^{0}$, and $p \bar{\Lambda} \pi^{-}$modes, respectively.

We require the mass of the baryon pair to be less than $2.85 \mathrm{GeV} / c^{2}$ for the study of the threshold enhancement effect. The $M_{\mathrm{bc}}$ distributions (with $|\Delta E|<0.05 \mathrm{GeV}$ ), and the $\Delta E$ distributions (with $M_{\mathrm{bc}}>5.27 \mathrm{GeV} / c^{2}$ ) for the $p \bar{p} K^{+}, p \bar{p} K_{S}^{0}$ and $p \bar{\Lambda} \pi^{-}$modes are shown in Fig. 2. The projections of the fit results are shown in Fig. 2 by solid curves. The $B$ yields are $217 \pm 17,28.6$ ${ }_{-5.8}^{+6.5}$, and $48.8{ }_{-7.5}^{+8.2}$ for the $p \bar{p} K^{+}, p \bar{p} K_{S}^{0}$, and $p \bar{\Lambda} \pi^{-}$modes, respectively. The measured branching fractions by summing the partial branching fractions in mass bins below $2.85 \mathrm{GeV} / c^{2}$ are $\mathcal{B}\left(B^{+} \rightarrow p \bar{p} K^{+}\right)=\left(4.59_{-0.34}^{+0.38} \pm 0.50\right) \times 10^{-6}$, $\mathcal{B}\left(B^{0} \rightarrow p \bar{p} K_{S}^{0}\right)=\left(1.04_{-0.19}^{+0.26} \pm 0.12\right) \times 10^{-6}$, and $\mathcal{B}\left(B^{0} \rightarrow p \bar{\Lambda} \pi^{-}\right)=\left(2.62_{-0.40}^{+0.44} \pm\right.$ $0.31) \times 10^{-6}$.

We study the proton angular distribution of the baryon-antibaryon pair system in its helicity frame. The angle $\theta_{p}$ is defined as the angle between the proton direction and the meson direction in the baryon-antibaryon pair rest frame. Note that the angle is determined by $\bar{p}$ and $K^{+}$(or $p$ and $K^{-}$) in the $p \bar{p} K^{+}$mode for definiteness. Fig. 3(a)-(c) shows the branching fractions as a function of $\cos \theta_{p}$. We define the angular asymmetry as $A=\frac{N_{+}-N_{-}}{N_{+}+N_{-}}$, where $N_{+}$and $N_{-}$stand for the efficiency corrected $B$ yield with $\cos \theta_{p}>0$ and $\cos \theta_{p}<0$, respectively. The angular asymmetry is determined to be $0.59_{-0.07}^{+0.08}$ for the $p \bar{p} K^{+}$mode. The asymmetry of the distribution indicates that the fragmentation picture is favored. Antiprotons are emitted along the $K^{+}$direction most of the time, which can be explained by a parent $\bar{b} \rightarrow \bar{s}$ penguin transition followed by $\bar{s} u$ fragmentation into the final state as shown in Fig. 4. The energetic $\bar{s}$ quark picks up the $u$ quark from a $u \bar{u}$ pair in vacuum and the remaining $\bar{u}$ quark then drags a $\bar{u} \bar{d}$ diquark out of vacuum. This simple picture can describe the $\bar{p}-K^{+}$angular correlation. The spectator $u$ quark and leftover $u d$ diquark form an proton.

The $\cos \theta_{p}$ distribution of the $p \bar{p} K_{S}^{0}$ mode can not support nor refute this fragmentation interpretation because of low statistics and no flavor information. The distribution for the $p \bar{\Lambda} \pi^{-}$mode is quite flat (i.e. in favor of the gluonic picture), in contrast to that of the $p \bar{p} K^{+}$mode, although both presumably share a common origin in the $\bar{b} \rightarrow \bar{s}$ transition. In fact, this parentage suggests that it would be useful to examine the proton angular distribution relative to the $\bar{\Lambda}$ direction in the $p \pi^{-}$rest frame. We remove the $M_{p \bar{\Lambda}}<2.85 \mathrm{GeV} / c^{2}$ constraint in order to check the full angular region; the result is shown in Fig. 3(d). It is evident that the fragmentation interpretation is supported: the proton tends to emerge parallel to the $\bar{\Lambda}$ baryon.

As a cross check, the distribution of $\cos \theta_{p}$ for background events in the $p \bar{p} K^{+}$ sample is shown in Fig. 3(e). Similar distributions are obtained for the backgrounds of the $p \bar{p} K_{S}^{0}$ and $p \bar{\Lambda} \pi^{-}$modes. The background has a $1+\alpha \cos ^{2} \theta_{p}$ distribution, which can be explained as arising from the random combina- 
tion of two high momentum particles from the $q \bar{q}$ jets. The continuum MC simulation also confirms this feature.

The newly observed narrow pentaquark state, $\Theta^{+}[19]$, can decay into $p K_{S}^{0}$. We perform a search in $B^{0} \rightarrow p \bar{p} K_{S}^{0}$ by requiring $1.53 \mathrm{GeV} / c^{2}<M_{p K_{S}^{0}}<1.55$ $\mathrm{GeV} / c^{2}$. The $M_{\mathrm{bc}}$ and $\Delta E$ projection plots in Fig. 5 show no evidence for a pentaquark signal. Since there are few events in the fit window, we fix the background shapes from sideband data. We use the fit results to estimate the expected background $(0.42 \pm 0.13)$ and compare this with the observed one event in the signal region to set an upper limit on the signal yield of 3.9 events at the $90 \%$ confidence level $[20,21]$. The systematic uncertainty is included in this limit. The related upper limit on the product of branching fractions is $\mathcal{B}\left(B^{0} \rightarrow \Theta^{+} \bar{p}\right) \times \mathcal{B}\left(\Theta^{+} \rightarrow p K_{S}^{0}\right)<2.3 \times 10^{-7}$. We also perform a search for $\Theta^{++}$, which can decay to $p K^{+}$in the mode $B^{+} \rightarrow p \bar{p} K^{+}[22]$. Because there are only theoretical conjectures for the existence of such a state, we examine the wider mass region of $1.6 \mathrm{GeV} / c^{2}<M_{p K^{+}}<1.8 \mathrm{GeV} / c^{2}$. We find no evidence for signal. Assuming this state is narrow and centered near 1.71 $\mathrm{GeV} / c^{2}$, the upper limit on the yield is 3.3 events at the $90 \%$ confidence level. The corresponding upper limit product of branching fractions is $\mathcal{B}\left(B^{+} \rightarrow\right.$ $\left.\Theta^{++} \bar{p}\right) \times \mathcal{B}\left(\Theta^{++} \rightarrow p K^{+}\right)<9.1 \times 10^{-8}$ at the $90 \%$ confidence level.

One theoretical conjecture [7] suggests that a possible glueball resonance $\mathcal{G}$ (e.g., $f_{J}(2220)$ [23]) with mass near $2.3 \mathrm{GeV} / c^{2}$ may contribute to the $M_{p \bar{p}}$ threshold peaking behavior for the $p \bar{p} K^{+}$mode. Since the $M_{p \bar{p}}$ mass resolution is about $10 \mathrm{MeV} / c^{2}$, we scan through the $2.2 \mathrm{GeV} / c^{2}<M_{p \bar{p}}<2.4 \mathrm{GeV} / c^{2}$ mass region with a $20 \mathrm{MeV} / c^{2}$ wide window. The largest upper limit on the yield is found to be 18.9 at $2.21 \mathrm{GeV} / c^{2}$. We use this data set to set an upper limit on the product of branching fractions of $\mathcal{B}\left(B^{+} \rightarrow \mathcal{G} K^{+}\right) \times \mathcal{B}(\mathcal{G} \rightarrow p \bar{p})<$ $4.1 \times 10^{-7}$ at the $90 \%$ confidence level for a narrow glueball state with mass in the $2.2-2.4 \mathrm{GeV} / c^{2}$ range. The theoretical expectation is around $1 \times 10^{-6}$.

In summary, using $152 \times 10^{6} B \bar{B}$ events, we measure the mass and the angular distributions of the baryon-antibaryon pair system near threshold for the $p \bar{p} K^{+}, p \bar{p} K_{S}^{0}$ and $p \bar{\Lambda} \pi^{-}$baryonic $B$ decay modes. A quark fragmentation interpretation is supported, while a gluonic resonant state picture is disfavored. Searches for a $B$ meson decaying into the $\Theta^{+}$pentaquark or a glueball in the above related modes give null results.

We thank the KEKB group for the excellent operation of the accelerator, the KEK cryogenics group for the efficient operation of the solenoid, and the KEK computer group and the National Institute of Informatics for valuable computing and Super-SINET network support. We acknowledge support from the Ministry of Education, Culture, Sports, Science, and Technology of Japan and the Japan Society for the Promotion of Science; the Australian Research Council and the Australian Department of Education, Science and Training; 
the National Science Foundation of China under contract No. 10175071; the Department of Science and Technology of India; the BK21 program of the Ministry of Education of Korea and the CHEP SRC program of the Korea Science and Engineering Foundation; the Polish State Committee for Scientific Research under contract No. 2P03B 01324; the Ministry of Science and Technology of the Russian Federation; the Ministry of Higher Education, Science and Technology of the Republic of Slovenia; the Swiss National Science Foundation; the National Science Council and the Ministry of Education of Taiwan; and the U.S. Department of Energy.

\section{References}

[1] K. Abe et al. (Belle Collaboration), Phys. Rev. Lett. 88, 181803 (2002).

[2] M.Z. Wang et al. (Belle Collaboration), Phys. Rev. Lett. 90, 201802 (2003).

[3] M.Z. Wang et al. (Belle Collaboration), Phys. Rev. Lett. 92, 131801 (2004).

[4] Y.J. Lee et al. (Belle Collaboration), Phys. Rev. Lett. 93, 211801 (2004).

[5] W.S. Hou and A. Soni, Phys. Rev. Lett. 86, 4247 (2001).

[6] C.K. Chua, W.S. Hou and S.Y. Tsai, Phys. Lett. B 528, 233 (2002).

[7] C.K. Chua, W.S. Hou and S.Y. Tsai, Phys. Lett. B 544, 139 (2002).

[8] J.L. Rosner, Phys. Rev. D 68, 014004 (2003).

[9] B. Kerbikov, A. Stavinsky, and V. Fedotov, Phys. Rev. C 69, 055205 (2004).

[10] J.Z. Bai et al. (BES Collaboration), Phys. Rev. Lett. 91, 022001 (2003); M. Ablikim et al. (BES Collaboration), Phys. Rev. Lett. 93, 112002 (2004).

[11] Throughout this report, inclusion of charge conjugate mode is always implied unless otherwise stated.

[12] S. Kurokawa and E. Kikutani, Nucl. Instr. and Meth. A 499, 1 (2003).

[13] A. Abashian et al. (Belle Collaboration), Nucl. Instr. and Meth. A 479, 117 (2002).

[14] R. Brun et al., GEANT 3.21, CERN Report No. DD/EE/84-1, 1987.

[15] R.A. Fisher, Annals of Eugenics 7, 179 (1936).

[16] K. Abe et al. (Belle Collaboration), Phys. Lett. B 517, 309 (2001).

[17] There are small corrections applied to these parameters based on the study of the difference between data and $\mathrm{MC}$ in $B \rightarrow D \pi$ decays.

[18] H. Albrecht et al. (ARGUS Collaboration), Phys. Lett. B 241, 278 (1990); ibid. B 254, 288 (1991). 
[19] See, e.g., T. Nakano et al. (LEPS Collaboration), Phys. Rev. Lett. 91, 012002 (2003).

[20] G.J. Feldman and R.D. Cousins, Phys. Rev. D 57, 3873 (1998).

[21] J. Conrad et al., Phys. Rev. D 67, 012002 (2003).

[22] T.E. Browder, I. Klebanov and D. Marlow, Phys. Lett. B 587, 62 (2004).

[23] S. Eidelman et al. (Particle Data Group), Phys. Lett. B 592, 594 (2004). 

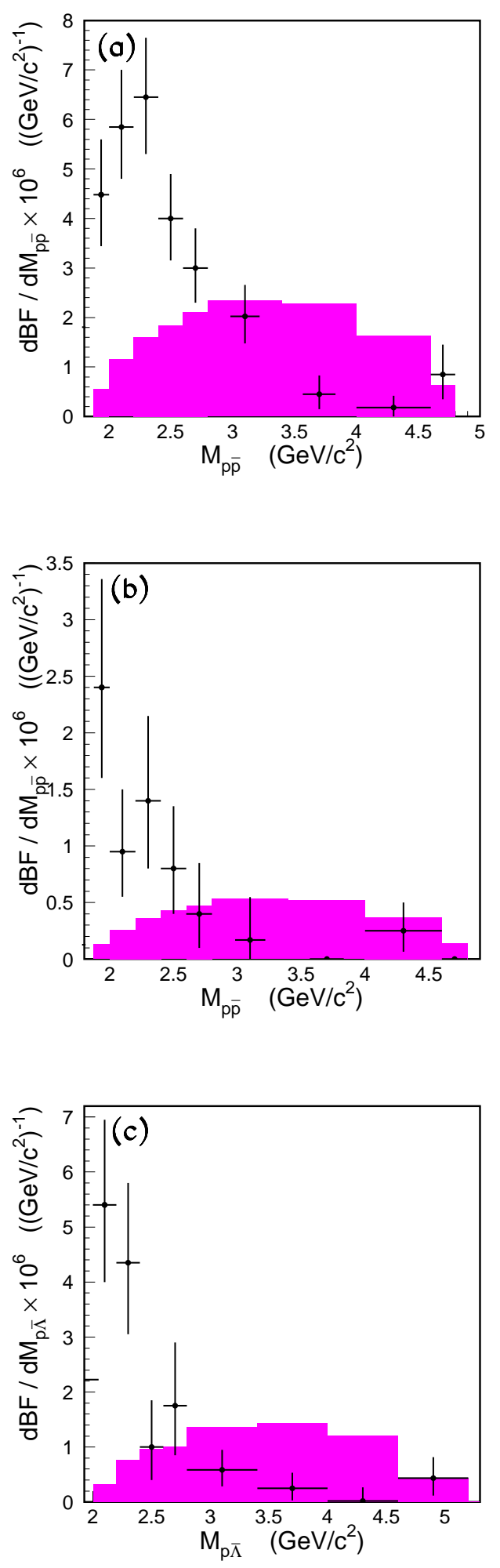

Fig. 1. Differential branching fraction for (a) $p \bar{p} K^{+}$, (b) $p \bar{p} K_{S}^{0}$, and (c) $p \bar{\Lambda} \pi^{-}$modes as a function of baryon-antibaryon pair mass. The shaded distribution shows the expectation from a phase-space MC simulation with area scaled to the signal yield. A charmonium veto has been applied in (a) and (b). 

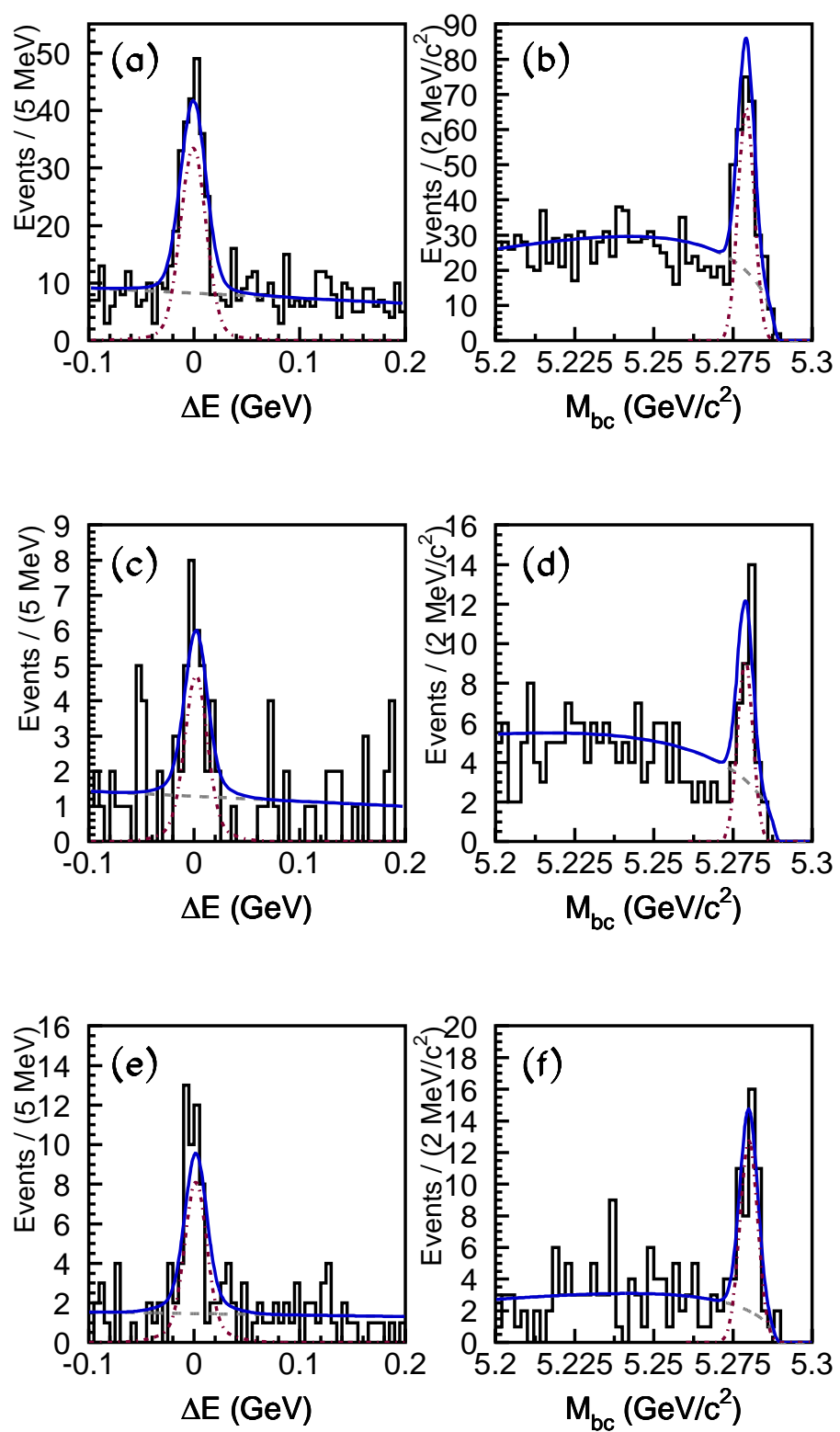

Fig. 2. Distributions of $\Delta E$ and $M_{\mathrm{bc}}$, respectively, for (a) and (b) $p \bar{p} K^{+}$, (c) and (d) $p \bar{p} K_{S}^{0}$, and (e) and (f) $p \bar{\Lambda} \pi^{-}$modes with baryon-antibaryon pair mass less than $2.85 \mathrm{GeV} / c^{2}$. The solid, dotted and dashed lines represent the combined fit result, fitted signal and fitted background, respectively. 

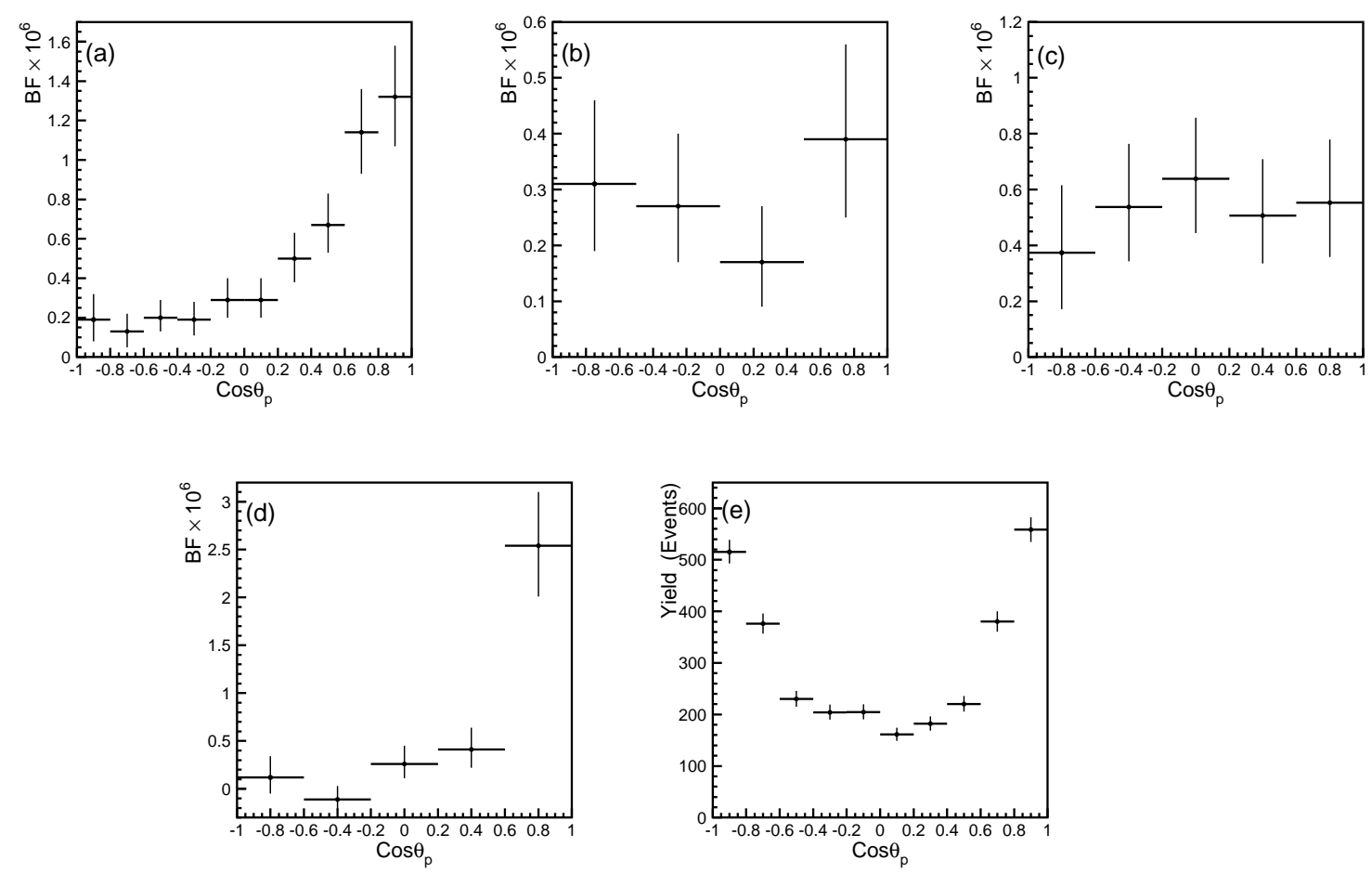

Fig. 3. Branching fraction vs. $\cos \theta_{p}$ in the baryon-antibaryon pair system for (a) $p \bar{p} K^{+}$, (b) $p \bar{p} K_{S}^{0}$, and (c) $p \bar{\Lambda} \pi^{-}$modes. (d) The proton angular distribution of the $p \pi^{-}$system against the $\bar{\Lambda}$ direction in the $p \bar{\Lambda} \pi^{-}$mode. (e) $p \bar{p} K^{+}$background yield vs. $\cos \theta_{p}$.

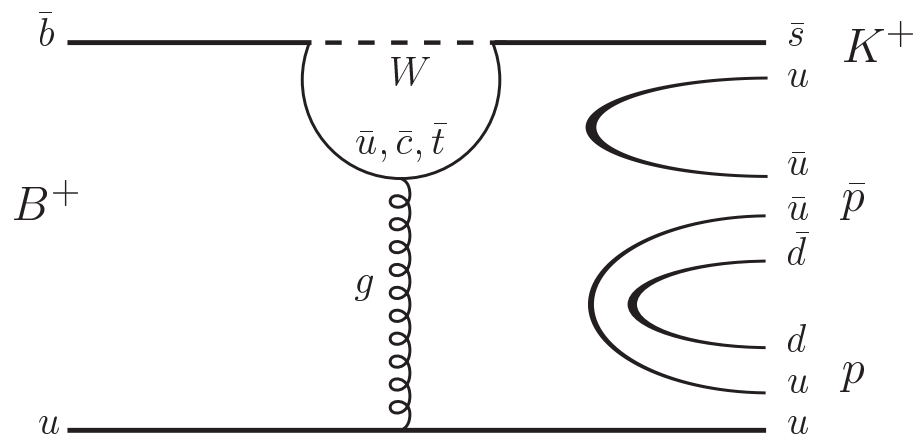

Fig. 4. Simple quark diagram for an illustration of the $B^{+} \rightarrow p \bar{p} K^{+}$decay. 


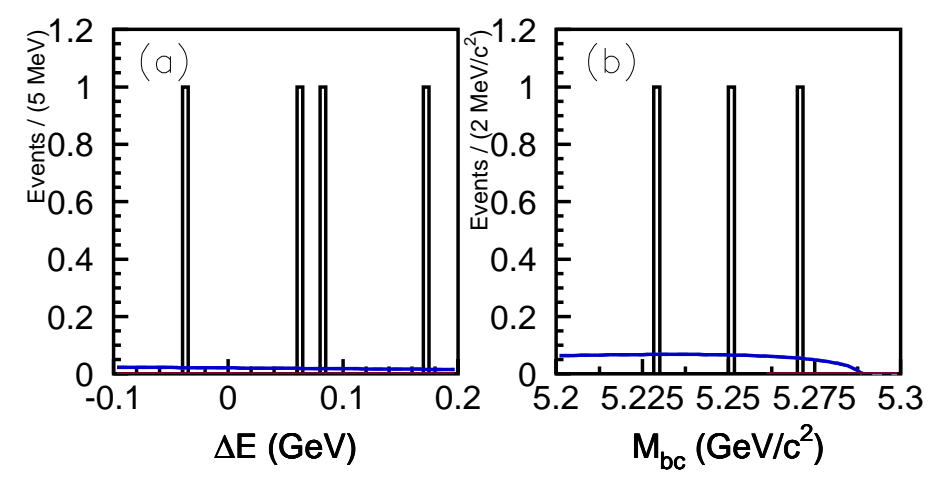

Fig. 5. Distributions of $M_{\mathrm{bc}}$ and $\Delta E$ for the $p \bar{p} K_{S}^{0}$ mode with 1.53 $\mathrm{GeV} / c^{2}<M_{p K_{S}^{0}}<1.55 \mathrm{GeV} / c^{2}$ in the region where the $\Theta^{+}$pentaquark is expected. The curves represent the fit projections. 